\title{
Participation and Activities of Daily Living, Cognition, And Motor Function According to Residential Area in Stroke Survivors
}

\author{
Eun-Young Park, PT, PhD • Won-Ho Kim, PT, $\mathrm{PhD}^{1 \dagger}$
}

\author{
Department of Secondary Special Education, College of Education, Jeonju University,
} ${ }^{1}$ Department of Physical Therapy, Ulsan College

Received: April 13, 2021 / Revised: April 13, 2021 / Accepted: May 11, 2021

(c) 2021 J Korean Soc Phys Med

\section{| Abstract |}

PURPOSE: Social participation is essential for stroke survivors. Although participation restrictions are affected by several factors, a few studies have focused on the effects of living in a particular residential area (urban versus rural) on the participation restrictions after a stroke. This study examined the factors affecting participation restrictions in stroke survivors according to the residential area.

METHODS: One hundred sixty-six stroke patients (including 130 living in urban areas and 36 living in rural areas) were recruited for this study. The Korean Modified Barthel Index (K-MBI) was used to assess the activities of daily living (ADL). The Korean Mini-Mental Examination Status (MMSE-K) was used to assess cognition. The motor function was evaluated using the Fugl-Meyer function assessment (FMA). Path analysis was used to test the hypothesized model of participation restriction.

RESULTS: The proposed path model showed good fit

$\dagger$ Corresponding Author : Won-Ho Kim whkim@uc.ac.kr, https://orcid.org/0000-0002-8984-8328

This is an Open Access article distributed under the terms of the Creative Commons Attribution Non-Commercial License (http://creativecommons.org/licenses/by-nc/3.0) which permits unrestricted non-commercial use, distribution, and reproduction in any medium, provided the original work is properly cited. indices. In rural and urban areas, the direct effects were significant between the ADL and participation restrictions ( $\beta$ $=-.673$ and -.457 , respectively). For urban areas, the direct effects were significant between cognition and participation restrictions $(\beta=-.252)$. In both area types, motor function and cognition had a significant direct effect on the ADL.

CONCLUSION: For urban community-dwelling stroke survivors, the ADL and cognition had direct effects on participation restrictions. For rural area stroke survivors, the ADL had direct effects on participation restrictions. Activity level exercise programs help reduce the participation restrictions. Moreover, it is essential to address cognition training to improve participation in urban communitydwelling stroke survivors.

Key Words: Activities of daily living, Participation, Path analysis, Stroke

\section{Introduction}

The incidence of stroke has decreased to $23.8 \%$ by controlling the risk factors and acute care over the last 10 years in Korea. On the other hand, the prevalence of stroke and the cost of stroke care have increased [1]. Individuals with stroke have a range of impairments, such 
as motor weakness, spasticity, sensory, and cognition deficiency [2]. One year after a stroke, approximately $33 \%$ of stroke survivors have a functional dependence on daily living activities, mobility, or speech [3]. The goal of rehabilitation after stroke is to recover their social role and participation [4]. Various interventions have been applied to achieve this goal. Eng and Tang [5] suggested using the International Classification of Functioning (ICF) model as a conceptual framework to improve the functional outcome. This is called the top-down approach focusing on activity-based intervention rather than impairment-based intervention. Furthermore, the ICF framework emphasizes contextual factors that represent the background of an individual's life and living. Bouffioulx, Arnould [6] suggested that satisfaction with activity and participation could not simply be inferred from the body functions and activities because it depends on complex interactions between functional, personal, and environmental factors. Robinson and Matsuda [7] reported that the environmental dimensions were associated with participation in community walking after a stroke. Hence, because participation in the community is affected by environmental factors, healthcare specialists should consider these factors when assessing and planning interventions for improving social participation after stroke.

Investigating the complex relationship between the person, environment, and participation and the impact of these factors on health and function are major rehabilitation research areas and challenges [8,9,10]. Environmental factors can help improve participation and alleviate the barriers that inhibit participation according to the individual's social role and context [11,12]. Although the International Classification of Functioning, Disability, and Health (ICF) has prompted a resurgence of efforts to measure the environmental factors in disability and rehabilitation research [13,14], there is insufficient evidence for the impact of environmental factors. Environmental modifications and system-level changes can help remove the participation barriers [15]. The individual function regarding participation in the community should be evaluated in the individual's environmental context. Although the effects of the geographic living area (urban versus rural) on participation restrictions have been reported, there is little evidence on the effects of residential areas on the participation of stroke survivors. Chau and Thompson [16] performed path analysis to verifying the determinants of participation restrictions among communitydwelling stroke survivors. They analyzed 188 stroke survivors 12 months after discharge in two rehabilitation hospitals in Hong Kong. Moreover, they reported that more depressive symptoms $(\beta=-.27)$, low state self-esteem ( $\beta$ $=.20)$, female sex $(\beta=.13)$, older age $(\beta=-.11)$, and living in a residential care facility $(\beta=-.12)$ have a direct effect on participation restrictions.

This study examined whether the activities of daily living (ADL) of stroke patients mediate the relationship between cognition and motor function and participation limitation. Five hypotheses were formulated and tested. First, it was hypothesized that cognition and motor function would be positively related to the ADL. Second, it was theorized that cognition and motor function would be negatively related to participation restriction. Third, a higher level of $\mathrm{ADL}$ would be negatively related to participation restriction. The fourth hypothesis is that the regression weights of cognition and motor function on participation restrictions would be insignificant. Fifth, the indirect and mediated effects of cognition and motor function on participation restrictions through the ADL would be significant. A previous study [17] suggested that professionals should pay attention to the role of physical and social environmental factors to help stroke patients return to social participation. The hypotheses were verified by accounting for the residential environments (urban and rural). 


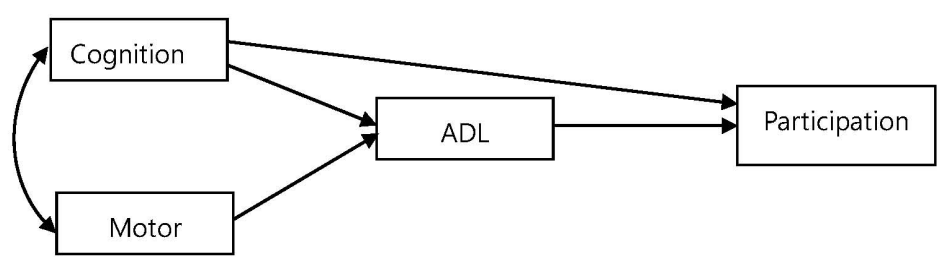

Fig. 1. Preliminary model.

\section{Methods}

\section{Participants and Data Collection}

A retrospective analysis of cross-sectional data was conducted to investigate the predictors of participation restrictions in chronic stroke survivors. The Ethics Review Board of Yonsei University approved this study. One hundred sixty-six stroke patients (109 men and 57 women) were analyzed. One-hundred thirty stroke patients lived in an urban community, and 36 lived in a rural community. They had been diagnosed with stroke based on the hospital records and experienced their first stroke. The exclusion criteria were cognitive dysfunction, as demonstrated by a score of $\leq 18$ on the Korean Mini-Mental State Examination (MMSE-K). Occupational therapists carried out the measurements. There were no incomplete responses. The recommended minimum sample size was a 5:1 ratio of the number of free parameters [18]. Six parameters were included in the present study, and the minimum sample size was 30 participants. The mean age of the 166 participants was 53.10 years $(\mathrm{SD}=13.47) ; 34.34 \%$ were women. Regarding the stroke diagnosis periods, the time ranged from 12 to 269 months with an average of 51.86 months ( $\mathrm{SD}=48.20$ ). The mean score for the MMSE-K was 25.75 ( $\mathrm{SD}=3.26$ ). Informed consent was obtained from all participants. The Ethics Committee of Yonsei University granted ethical approval for this study.

\section{Measurements}

The cognition was measured using the MMSE-K. In this study, Cronbach's a was .83. A Fugl-Meyer Function
Assessment (FMA) was used to measure the motor performance [19]. The maximum scores of the upper and lower extremities were 66 and 34, respectively. In this study, Cronbach's a was .98 . The activities of daily living were measured using the Korean version of the modified Barthel index (K-MBI) [20]. The K-MBI evaluates 10 different areas of $\mathrm{ADL}$, including feeding, transfer, mobility, ascending and descending stairs, and bowel and bladder control. The impact on participation and autonomy (IPA) was used to measure participation restrictions [21]. IPA consists of 32 items with five dimensions. A lower score means higher participation and autonomy. In this study, Cronbach's a was .95 .

\section{Statistical Analysis}

This study proposed a path model to examine the causal relationships among participation, activities of daily living, cognition, and motor impairment of stroke patients according to residential areas. Fig. 1 presents the preliminary model. Path analysis is a statistical technique that uses both bivariate and multiple linear regression techniques to test the causal relationships among the variables in a specialized model [22]. The path coefficients were computed using multiple regression analyses based on the hypothesized model; the colinearity of the data was analyzed using collinearity diagnostics in SPSS. The AMOS 17.0 statistical program was used to analyze the path models. Root mean square error of approximation (RMSEA) values $<.05, .06-.08, .08-.10$, and $>.1$ indicate good, reasonable, mediocre, and poor fit, respectively. The normed fit index (NFI) and comparative fit index (CFI) $>$ .90 also indicated a good fit [23]. 
Table 1. Baseline Data of MMSE-K, Fugl-Myer, ADL, and Participation Limitation

\begin{tabular}{cccc}
\hline & Mean & SD & Median (25th / 75th percentiles) \\
\hline MMSE-K $^{\mathrm{a}}$ & 27.22 & 3.26 & $25.75 / 30.00$ \\
Fugl-Myer & 56.80 & 27.21 & $36.50 / 81.25$ \\
MBI-K $^{\mathrm{b}}$ & 81.90 & 18.64 & $87.00 / 96.00$ \\
IPA $^{\mathrm{c}}$ & 60.72 & 30.60 & $40.75 / 82.00$ \\
\hline
\end{tabular}

${ }^{a}$ Korean version of the Mini-Mental Examination

borean version of the Modified Barthel Index

'Impact on Participation and Autonomy

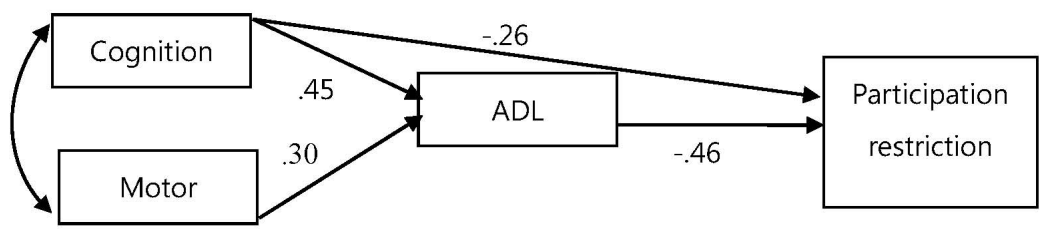

Fig. 2. Proposed path model in urban communities.

\section{Result}

\section{Baseline Data}

Table 1 lists the baseline data for MMSE-K, Fugl-Myer, KMBI, and IPA.

\section{Multicollinearity Test}

The inter-correlations of motor function, cognition, $\mathrm{ADL}$, and participation limitation were analyzed using the Pearson correlation coefficients. Three variables are found to be significant $(\mathrm{p}<.001)$. Multicollinearity was not detected because the bivariate correlations did not exceed .80 [24].

\section{Preliminary Path Analysis}

Preliminary path analyses were conducted to verify the significant variables for model identification. The analysis for stroke residents in urban areas was performed. The probability of receiving a critical ratio as large as 2.276 in the absolute value was significant from cognition to $\operatorname{ADL}(\beta=.452 ; p<.001)$ and from motor function to ADL $(\beta=.298 ; p<.001)$. The regression weights for
ADL in the prediction of participation limitations $(\beta=$ $-.454 ; \mathrm{p}<.001)$, and for cognition in the prediction of participation limitations $(\beta=-.251 ; \mathrm{p}=.002)$ were significantly different from zero at the .05 level. The regression weight for motor function $(\beta=-.008 ; p=.917)$ in predicting participation limitations was not significantly different. The proposed path model for the urban area was constructed by deleting the insignificant path from the motor function to ADL in the preliminary model (Fig. 2).

An analysis for stroke residents in rural areas was then performed. The probability of obtaining a critical ratio as large as 2.276 in the absolute value was significant from cognition to $\mathrm{ADL}(\beta=.425 ; \mathrm{p}<.001)$ and from motor function to $\mathrm{ADL}(\beta=.389 ; \mathrm{p}<.001)$. The regression weight for $\mathrm{ADL}$ in predicting participation limitations $(\beta=-.656$; $\mathrm{p}<.001)$ was significantly different from zero at the .05 level. The regression weight for cognition $(\beta=.096 ; p=$ $.491)$ and motor function $(\beta=-.008 ; p=.917)$ in predicting participation limitations was not significantly different. The proposed path model for the rural areas was constructed by deleting the insignificant path from the motor function 


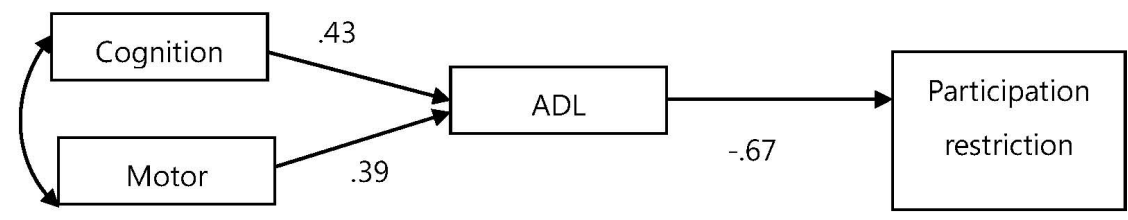

Fig. 3. Proposed path model in rural communities.

Table 2. Estimates of the Regression Weights of the Proposed Model

\begin{tabular}{ccccccc}
\hline Residential area & Path & $\mathrm{B}^{\mathrm{a}}$ & $\beta^{\mathrm{b}}$ & S.E. $^{\mathrm{c}}$ & C.R. $^{\mathrm{d}}$ & $\mathrm{p}$ \\
\hline Urban & $\mathrm{ADL} \leftarrow$ Cognition & 2.59 & .45 & .42 & 6.16 & $<.001$ \\
& $\mathrm{ADL} \leftarrow$ Motor & .204 & .30 & .05 & 4.07 & $<.001$ \\
& Participation restriction $\leftarrow$ ADL & -.75 & -.46 & .13 & -5.58 & $<.001$ \\
\multirow{2}{*}{ Rural } & Participation restriction $\leftarrow$ Cognition & -2.63 & -.25 & .72 & -3.07 & .002 \\
& ADL $\leftarrow$ Cognition & 1.90 & .43 & .60 & 3.16 & .002 \\
& ADL $\leftarrow$ Motor & 2.44 & .39 & .08 & 2.90 & .004 \\
& Participation restriction $\leftarrow$ ADL & -.95 & -.67 & .18 & -5.38 & $<.001$ \\
\hline
\end{tabular}

aunstandardized coefficients

bstandardized coefficients

cstandard error

${ }^{\mathrm{d}}$ critical ratio

to the $\mathrm{ADL}$ in the preliminary model (Fig. 3).

\section{Model Fit}

The proposed model showed excellent fit indices in each group. RMSEA scores $<.05$ indicated good and acceptable model fits, as did NFI and CFI scores $>.95$.

\section{Direct and Indirect Effect of Variables}

Table 2 lists the estimated regression weights of the proposed model, and Table 3 presents the direct and indirect effects of the variables.

The regression weights and direct and indirect effects in urban areas were followed. The cognition and motor function affect the ADL directly and a significant indirect effect on participation limitations through the ADL. The direct paths from cognition to participating in the ADL was $-.252(\mathrm{p}=.002)$. Cognition and motor function accounted for $38.5 \%$ of the variance in explaining the $\mathrm{ADL}$; cognition, motor function, and the ADL accounted for $40.0 \%$ of the variance in participation limitations. The standardized direct effects of cognition on the ADL were .452 , and the standardized direct effects of motor function on the ADL were .298. The standardized direct effect of cognition and the ADL on participation limitations was -.252 and -.457 , respectively. The standardized indirect effects of motor function and cognition on participation limitations were -.207 and -.136 , respectively. The regression weights in rural areas were analyzed. The path coefficient from cognition to the ADL was $.425(p=.002)$ and $.389(\mathrm{p}=.004)$ from motor function to the ADL. The path coefficient from ADL to participation limitations was $-.673(\mathrm{p}<.001)$. Cognition and motor function accounted for $37.8 \%$ of the variance in explaining the $\mathrm{ADL}$; cognition, motor function, and $\mathrm{ADL}$ accounted for $45.3 \%$ of the 
Table 3. Direct and Indirect Effects of Variables

\begin{tabular}{|c|c|c|c|c|c|c|}
\hline Residential area & Predictor variables & Dependent Variables & Total Effect & Direct Effect & Indirect Effect & $\mathrm{R}^{2}$ \\
\hline \multirow[t]{5}{*}{ Urban } & Cognition & \multirow{2}{*}{ ADL } & $.45^{*}$ & $.45^{*}$ & & \multirow{2}{*}{.39} \\
\hline & Motor & & $.30 *$ & $.30 *$ & & \\
\hline & Cognition & \multirow{3}{*}{ Participation restriction } & $-.46^{*}$ & $-.25^{*}$ & $-.21 *$ & \\
\hline & Motor & & $-.14^{*}$ & & $-.14^{*}$ & .40 \\
\hline & ADL & & $-.46^{*}$ & $-.46^{*}$ & & \\
\hline \multirow[t]{5}{*}{ Rural } & Cognition & \multirow{2}{*}{$\mathrm{ADL}$} & $.43 *$ & $.43^{*}$ & & \multirow{2}{*}{.38} \\
\hline & Motor & & $.39 *$ & $.39 *$ & & \\
\hline & Cognition & \multirow{3}{*}{ Participation restriction } & $-.29 *$ & & $-.29 *$ & \multirow{3}{*}{.45} \\
\hline & Motor & & $-.26^{*}$ & & $-.26^{*}$ & \\
\hline & $\mathrm{ADL}$ & & $-.67^{*}$ & $-.67 *$ & & \\
\hline
\end{tabular}

variance in participation limitations. The standardized direct effect of cognition and motor function on the ADL was .425 and .389 , respectively. The standardized direct effect of ADL on participation limitations was -.673. The standardized indirect effects of motor function and cognition on participation limitations were -.286 and -.262 , respectively.

\section{Discussion}

Although there were no differences in improved functional outcomes between urban and rural patients during the inpatient phase [25], it was anticipated that the degree of social participation of stroke survivors might differ after discharge according to the residential area. This study investigated the factors contributing to participation restrictions according to the living environment in people with chronic stroke. According to ICF concepts, the ADL represent a limitation of activity at the personal level, whereas participation restrictions are a limitation at the social level. In this study, cognition and motor function were positively correlated with the ADL and negatively correlated with participation restrictions. Furthermore, the ADL were negatively associated with participation restriction. Participation restrictions had a more significant correlation with the $\mathrm{ADL}$ and cognition than with motor function. These results were consistent with those of previous studies [4,26]. Van Der Zee and Visser-Meily [27] emphasized physical and cognitive independence for participation after stroke. Physical and cognitive issues among individual barriers were identified most frequently and are often pointed to as the primary reason for non-participation [15]. The effect of motor and cognition on participation was significant, but the effect was different according to the residential area. For rural communitydwelling people with stroke, the path model proposed here indicates that the path coefficients show that the ADL $(\beta$ $=-.673$ ) have the most significant direct effect, explaining the largest variance in participation restriction. Moreover, the motor function $(\beta=-.286)$ and cognition $(\beta=-.262)$ had an indirect effect on participation restrictions through their effects on the ADL. The proposed path model shows that the motor function and cognition had a significant direct effect on the ADL. For urban community-dwelling people with stroke, unlike in rural cases, the path model proposed in this paper shows that the ADL $(\beta=-.457)$ and cognition $(\beta=-.252)$ affect participation restrictions directly. In addition, the motor function $(\beta=.298)$ had 
an indirect effect on participation restrictions through its effects on the ADL. Previous studies reported that the functional activity was a major factor contributing to participation restrictions. Chau and Thompson [16] reported that the path coefficients for the functional ability had the most significant direct effect on participation restrictions $(\beta=.51)$. Choi, Park $[28]$ reported that the ADL were important for directly affecting participation restrictions $(\beta$ $=-.43)$. In this study, the ADL related to the activity level in the ICF framework had the largest direct effect on participation restrictions. On the other hand, the path coefficients differed according to the living area. The path coefficients in urban and rural areas were -.457 and -.653 , respectively. This was attributed to the characteristics of rural areas. The functional ability of stroke survivors in rural areas was more important than that in urban areas because a rural area has poor environmental conditions (e.g., lack of public transport and facilities) for locomotion [29,30]. Zhang and Yan [31] reported that physical and service barriers were dominant barriers to the activity and participation and stroke survivors in the rural areas of China. Therefore, intervention programs need to focus on the activity to reduce participation restrictions, especially in rural areas.

Many researchers studied psychological factors affecting participation restriction. Chau and Thompson [16] reported that more depressive symptoms and low self-esteem directly affect participation restrictions. Zhang and Yan [31] reported that depression and social participation are inversely related. Other studies reported that self-efficacy [32] and hopeful thinking [28] could significantly predict important participation restrictions in people with stroke. This suggests that emotion regulation may be an essential factor for improving social participation. One study found that emotion control was a significant factor in reducing participation restriction after stroke [33]. Thus, recent studies focused on self-management programs for stroke survivors to improve the functional ability and participation
[34,35]. Cognition is essential for participating in a successful self-management program. Cognition function includes impulse control, planning, attention, and working memory [36]. Ayerbe and Ayis [37] suggested that the development of post-stroke depression was associated with cognitive impairment. Cognition was strongly associated with the executive function and motor function [38] and participation restriction [27].

In this study, cognition had a direct effect on the ADL and participation restrictions in urban areas. This suggests that cognition is considered an important factor for social participation after stroke. Fortunately, as cognitive functions respond positively to exercise [36], cognition and exercise training are necessary to improve cognition after stroke. The results also support the notion that more depressive symptoms and low state self-esteem directly affect participation restrictions. In the case of the urban area, stroke survivors may experience a sense of alienation and isolation because of the social structure of youth-oriented society. Cognition can be important because people with stroke have a functional role in a complex social structure. Hammel and Jones [15] reported that most environments did not support cognitive access, such as environmental aids or support for orientation, navigation, memory, and problem solving. The cognitive need for urban-dwelling stroke survivors indicates that environmental factors should be considered to improve participation level.

This study had some limitations. First, although the minimum sample size of this study was 30 participants, the relative sample size of stroke survivors from rural areas was small compared to that of the urban-dwelling stroke survivors. Additionally, the participants had $>18$ score of MMSE-K and various onset times. Thus, these findings are difficult to generalize to all people with stroke. Second, people with stroke need time to understand and adjust to their changed bodies and behaviors in the community [39]. Although the community-dwelling time after discharge may have contributed to these findings, this study did not 
investigate the duration of community-dwelling after discharge from the hospital. The ADL and cognition accounted for $40 \%$ of the variance in explaining participation restrictions in urban areas. The amount of variance explained in this study suggests that other variables, such as the community-dwelling time and social support, should be included to explain the participation restrictions.

\section{Conclusion}

Functional ability and cognition affected participation restrictions directly in urban community-dwelling stroke survivors, and only functional ability affected participation restrictions directly in rural areas. Thus, activity-level exercise programs help to reduce participation restrictions. In particular, it is important to address cognition training or decreasing cognitive needs to improve participation in urban community-dwelling stroke survivors.

\section{References}

[1] Stroke CRCf. Clinical practice guidelines for stroke. Seoul: Clinical Research Center for Stroke. Seoul: Clinical Research Center for Stroke. 2013.

[2] O'Sullivan S. Stroke In: O'Sullivan SB and Schmitz TJ. Physical Rehabilitation FA Davis, Philadelphia, PA. 2007:706-76.

[3] Murray CJ, Lopez AD, Organization WH. The global burden of disease: a comprehensive assessment of mortality and disability from diseases, injuries, and risk factors in 1990 and projected to 2020. Cambridge: Harvard University Press. 1996.

[4] Fride Y, Adamit T, Maeir A, et al. What are the correlates of cognition and participation to return to work after first ever mild stroke? Top Stroke Rehabil. 2015; 22(5):317-25.

[5] Eng JJ, Tang P-F. Gait training strategies to optimize walking ability in people with stroke: a synthesis of the evidence. Exp Rev Neurotherap. 2007;7(10):1417-36.

[6] Bouffioulx É, Arnould C, Thonnard J-L. Satisfaction with activity and participation and its relationships with body functions, activities, or environmental factors in stroke patients. Arch Phys Med Rehabil. 2011;92(9): 1404-10.

[7] Robinson CA, Matsuda PN, Ciol MA, et al. Participation in community walking following stroke: the influence of self-perceived environmental barriers. Phys Ther. 2013;93(5):620-27.

[8] Hammel J, Magasi S, Heinemann A, et al. Environmental barriers and supports to everyday participation: a qualitative insider perspective from people with disabilities. Arch Phys Med Rehabil. 2015;96(4):578-88.

[9] Magasi S, Hammel J, Heinemann A, et al. Participation: a comparative analysis of multiple rehabilitation stakeholders' perspectives. J Rehabil Med. 2009;41(11): 936-44.

[10] Mishler E. Validation in inquiry-guided research: The role of exemplars in narrative studies. Harvard Educ Rev. 1990;60(4):415-43.

[11] Heinemann AW, Lai J-S, Magasi S, et al. Measuring participation enfranchisement. Arch Phys Med Rehabil. 2011;92(4):564-71.

[12] Gray DB, Hollingsworth HH, Stark SL, et al. Participation survey/mobility: psychometric properties of a measure of participation for people with mobility impairments and limitations. Arch Phys Med Rehabil. 2006;87(2): 189-97.

[13] Organization WH. International classification of functioning, disability and health: ICF. Geneva: World Health Organization. 2001.

[14] Noonan VK, Kopec JA, Noreau L, et al. A review of participation instruments based on the International Classification of Functioning, Disabili and Health. Disabilil Rehabil. 2009;31(23):1883-901.

[15] Hammel J, Jones R, Gossett A, et al. Examining barriers and supports to community living and participation after 
a stroke from a participatory action research approach.

Top Stroke Rehabil. 2006;13(3):43-58.

[16] Chau JP, Thompson DR, Twinn S, et al. Determinants of participation restriction among community dwelling stroke survivors: a path analysis. BMC Neurol. 2009; 9(1):49.

[17] Jellema S, van Hees S, Zajec J, et al. What environmental factors influence resumption of valued activities post stroke: a systematic review of qualitative and quantitative findings. Clin Rehabil. 2017;31(7):936-47.

[18] Jackson DL. Sample size and number of parameter estimates in maximum likelihood confirmatory factor analysis: A Monte Carlo investigation. Struct Equat Model. 2001;8(2):205-23.

[19] Sanford J, Moreland J, Swanson LR, et al. Reliability of the Fugl-Meyer assessment for testing motor performance in patients following stroke. Phys Ther. 1993;73(7):447-54.

[20] Jung HY, Park BK, Shin HS, et al. Development of the Korean version of Modified Barthel Index (K-MBI): multi-center study for subjects with stroke. J Korean Acad Rehabil Med. 2007;31(3):283-97.

[21] Cardol M, de Haan RJ, van den Bos GA, et al. The development of a handicap assessment questionnaire: the Impact on Participation and Autonomy (IPA). Clin Rehabil. 1999;13(5):411-19.

[22] Olobatuyi ME. A user's guide to path analysis. University Press of America. 2006.

[23] Bentler PM. Comparative fit indexes in structural models. Psych Bull. 1990;107(2):238.

[24] Jobson J. Multiple linear regression: Applied multivariate data analysis. Springer. 1991.

[25] Manimmanakorn N, Vichiansiri R, Nuntharuksa C, et al. Quality of life after stroke rehabilitation among urban vs. rural patients in Thailand. J Med Assoc Thailand. 2011;91(3):394.

[26] Spitzer J, Tse T, Baum CM, et al. Mild impairment of cognition impacts on activity participation after stroke in a community-dwelling Australian cohort. Occup Particip Health. 2011;31(suppl):S8-15.

[27] Van Der Zee CH, Visser-Meily JM, Lindeman E, et al. Participation in the chronic phase of stroke. Top Stroke Rehabil. 2013;20(1):52-61.

[28] Choi YI, Park JH, Jung M-Y, et al. Psychosocial predictors of participation restriction poststroke in Korea: A path analysis. Rehabili Psych. 2015;60(3):286.

[29] Crowther MR, Scogin F, Johnson Norton M. Treating the aged in rural communities: the application of cognitivebehavioral therapy for depression. J Clin Psych. 2010;66(5):502-12.

[30] Kang H, Park K. Comparison of correlates of depression in late-life between urban and rural areas. J Korean Gerontol Soc. 2012;32(1):129-43.

[31] Zhang L, Yan T, You L, et al. Barriers to activity and participation for stroke survivors in rural China. Arch Phys Med Rehabil. 2015;96(7):1222-28.

[32] French MA, Moore MF, Pohlig R, et al. Self-efficacy mediates the relationship between balance/walking performance, activity, and participation after stroke. Top Stroke Rehabil. 2016;23(2):77-83.

[33] Cooper CL, Phillips LH, Johnston M, et al. The role of emotion regulation on social participation following stroke. British J Clin Psych. 2015;54(2):181-99.

[34] Warner G, Packer T, Villeneuve M, et al. A systematic review of the effectiveness of stroke self-management programs for improving function and participation outcomes: self-management programs for stroke survivors. Disabil Rehabil. 2015;37(23):2141-63.

[35] Cadilhac DA, Kilkenny MF, Srikanth V, et al. Do cognitive, language, or physical impairments affect participation in a trial of self-management programs for stroke? Intern J Stroke. 2016;11(1):77-84.

[36] Guiney H, Machado L. Benefits of regular aerobic exercise for executive functioning in healthy populations. Psychon Bul Rev. 2013;20(1):73-86.

[37] Ayerbe L, Ayis S, Wolfe CD, et al. Natural history, 
predictors and outcomes of depression after stroke: systematic review and meta-analysis. British J Psych. 2013;202(1):14-21.

[38] Walker C, Sunderland A, Sharma J, et al. The impact of cognitive impairment on upper body dressing difficulties after stroke: a video analysis of patterns of recovery. J Neurol Neurosurg Psych. 2004;75(1):43-8.

[39] Pallesen H. Body, coping and self-identity. A qualitative 5-year follow-up study of stroke. Disabil Rehabil. 2014;36(3):232-41. 